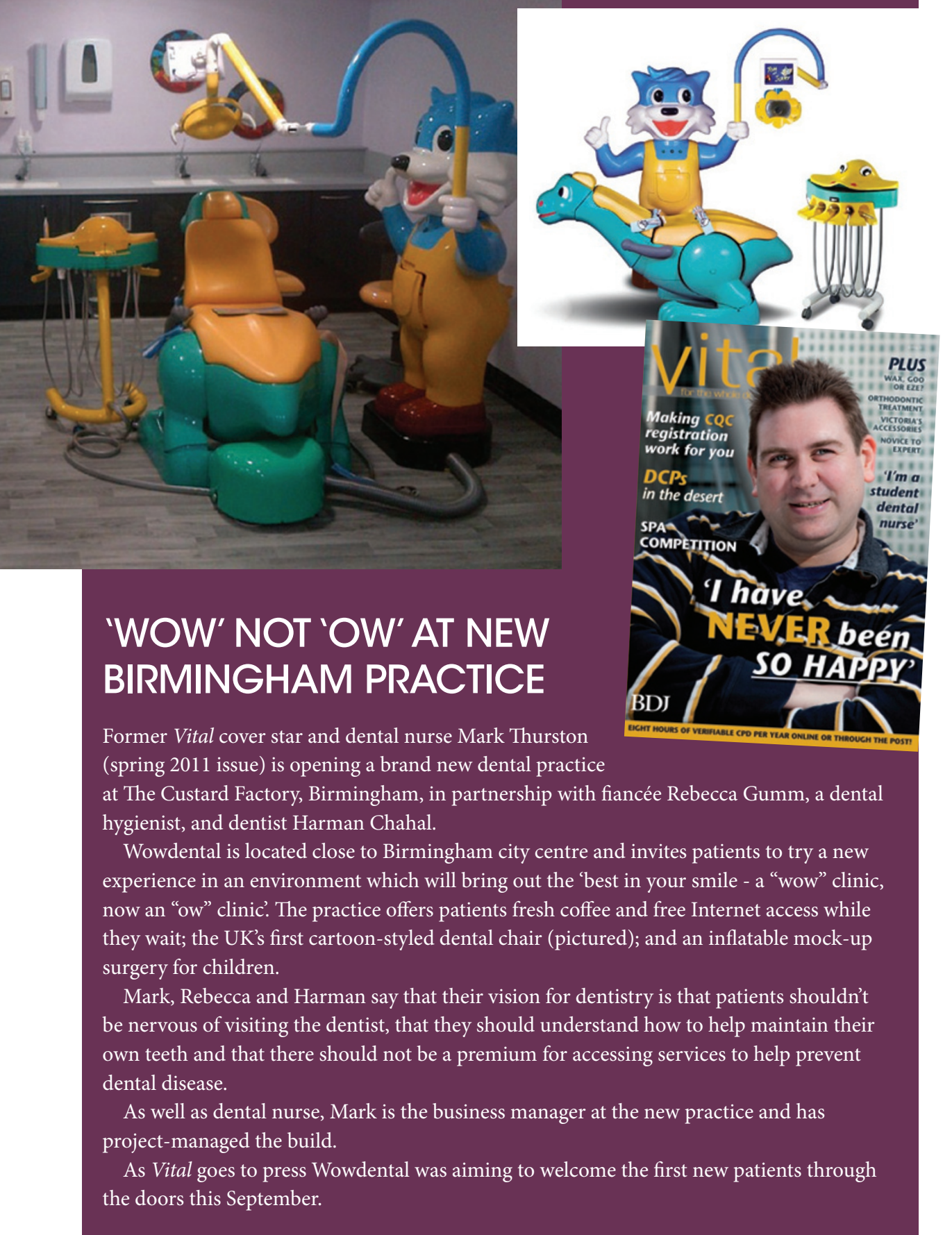

\section{'WOW' NOT 'OW' AT NEW}

Former Vital cover star and dental nurse Mark Thurston

at The Custard Factory, Birmingham, in partnership with fiancée Rebecca Gumm, a dental gienist, and dentist Harman Chahal.

experience in an environment which will bring out the "best in your smile - a "wow" clinic, now an "ow" clinic'. The practice offers patients fresh coffee and free Internet access while they wait; the UK's first cartoon-styled dental chair (pictured); and an inflatable mock-up argery for children. own teeth and that there should not be a premium for accessing services to help prevent dental disease.

roject-managed the build.

he doors this September.

\section{STINKY BREATH WORSE THAN STINKY FEET}

Bad breath has been named the third worst bad smell in Britain. A survey of over 2,000 people carried out for a UK-wide waste disposal company revealed that the worst smell as voted for by the British public is babies' nappies, followed by sewers on a hot day.

The complete top ten worst smells are:

Baby poo

Sewers on a hot day

3 Bad breath

4 Wet dog

5 Flatulence

Human sweat

7 Rotten food

8 Smoking

9 Smelly feet

10 The 'juice' at the bottom of your bin.

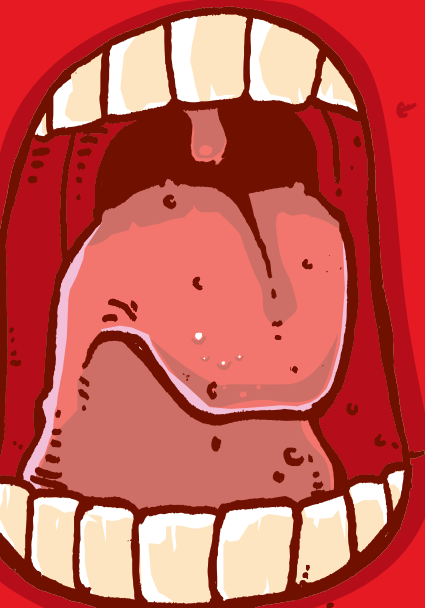

\title{
HYGIENISTS PRESENT TO YOUNG DENTISTS AT FULLY-BOOKED WORKSHOPS
}

Dental hygienist Shaun Howe and dental hygienist and therapist Amanda Gallie were thrilled to be invited to present a series of workshops both to other dental care professionals (DCPs) and to recently qualified dentists at this year's British Dental Conference and Exhibition in London.

The workshops, 'May the force be with you ... de-mystifying periodontal assessment (screenings)', were fully booked months in advance and over-subscribed and saw a wide range of practitioners from all areas of practice in attendance.

The sessions explored probing the gingivae while carrying out periodontal charting, its importance in record keeping, calibrating probing force and the use of ultrasonic scalers.

Shaun spoke to Vital about their experience at the event:
'We had some nerves going into the presentations; the normal ones for anyone involved in speaking such as "are my facts correct?" and "will they enjoy it?" but these things soon wash away once the delegates arrive.

'The groups were limited to 15 or so people so that during the hands-on sessions, delegates could receive our individual attention. Too

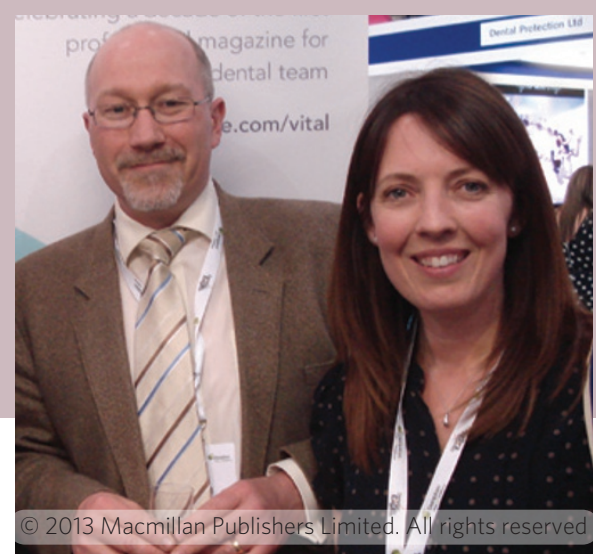

many presentations can be "death by slides" so we tried to keep slides to a minimum and focus on interaction.'

Shaun and Amanda were grateful for the support they received during the conference from the British Dental Association (BDA) and from Optident and EMS. Shaun said: 'Any DCP thinking of making a pitch to present at the Conference can do so with the sound knowledge that they will receive tremendous support in all aspects. Information is provided well in advance and allows the speakers to prepare well and there are many support staff available to help on the day?

Shaun and Amanda also took the time to join the celebration for Vital's tenth anniversary on the BDJ/Vital stand where Shaun met fellow members of the Vital Reader Panel. 